\title{
Enhanced Flip-FBMC visible light communication model
}

\author{
Abdullah Ali Qasim ${ }^{1}$, Husam Noman Mohammedali ${ }^{2}$, M. F. L. Abdullah ${ }^{3}$, R. Talib ${ }^{4}$, \\ Haidar Zaeer Dhaam ${ }^{5}$ \\ 1,3,4 Department Communication Engineering, University Tun Hussein Onn Malaysia, Johor, Malaysia \\ ${ }^{1,2,5}$ Eng. Technical College-Najaf, Al-Furat Al-Awsat Technical University, Iraq
}

\begin{tabular}{|c|c|}
\hline Article Info & ABSTRACT \\
\hline Article history: & \multirow{12}{*}{$\begin{array}{l}\text { VLC technology is one of the most promising technologies in the generations } \\
\text { of communications, especially 5G after the significant development of } \\
\text { optical semiconductors, specific laser diode, and the use of FBMC } \\
\text { modulation in the system. DCO-FBMC modulation surpasses all the } \\
\text { disadvantages of DCO-OFDM modulation techniques, as it is characterized } \\
\text { by high speed and large bandwidth, but this technique consumes high energy } \\
\text { when converting a real bipolar signal into a positive unipolar by adding DC- } \\
\text { bias. Besides the challenge of illumination distribution indoor typical room, } \\
\text { where the drop of the illumination appears in the middle of the room, besides } \\
\text { the power consumption. The Flip-FBMC technique was proposed to solve } \\
\text { this challenge, which helps convert the bipolar signal into unipolar by } \\
\text { separating the positive part from the negative part and converting it into } \\
\text { positive and merging it using Multiplexer and then sending the signal. } \\
\text { Besides this, the authors presented in this paper a new lighting model with } 5 \\
\text { luminescent units, using LD instead of LED. The Flip-FBMC profile gave } \\
\text { better energy savings and BER performance than DCO-FBMC, besides a } \\
\text { lighting model that covers dark areas and gives high SNR for the blind area } \\
\text { and give save power } 13.14 \% \text { from consumed power. }\end{array}$} \\
\hline Received Feb 2, 2021 & \\
\hline Revised Aug 2, 2021 & \\
\hline Accepted & \\
\hline Keywords: & \\
\hline DCO-FBMC & \\
\hline Flip-FBMC & \\
\hline Laser diode & \\
\hline LED & \\
\hline Optical power & \\
\hline SNR & \\
\hline Visible light communication & \\
\hline
\end{tabular}

This is an open access article under the CC BY-SA license.

\section{Corresponding Author:}

\author{
Abdullah Ali Qasim \\ Department Communication Engineering \\ University Tun Hussein Onn Malaysia \\ Parit Raja, Johor, Malaysia \\ Email: alzubydea@gmail.com, alzubydea@atu.edu.iq
}

\section{INTRODUCTION}

Communications are the major focus of information transmission, and as a result of the significant development, portable devices have increased the need for higher data rates, transmission efficiency, and bandwidth usage [1]. Here, the choice of modulation and effective demodulation must be vital in improving the effectiveness and reliability of the signal. The real challenge appears as wireless communication has become a very important technology, and one of the most important communication methods is very important is the visible light communication (VLC) [2]-[4] The VLC system uses solid-state optical semiconductors such as light-emitting diodes (LED) [5] and lasers diode (LD) [6], [7] that are used to achieve lighting and data transmission. VLC has shown that it is a major part of existing $5 \mathrm{G}$ networks. VLC providing wide bandwidth density solves the increasing high-bandwidth demand problem face $\mathrm{d}$ by RF-based networks [8]. These features and advantages present in the VLC system, make it a promising technology for the $5 \mathrm{G}$ system as it comes with many new challenges that open new research topics [9].

The limited bandwidth is one of the major problems facing the VLC system, so choosing the appropriate modulation is very important [10]-[12]. It must be highly efficient, as many modulations methods 
have been used, the most prominent of which is the OFDM system, the real signal and to achieve this, hermitian symmetry (H.S) is used [13]-[16]. The signal must be unipolar, so the OFDM scheme uses DC-bias to make the signal positive. There are other types of ways to make the signal unipolar, but each scheme has positives and negatives, but there are major weaknesses that OFDM modulation suffers from as it suffers from narrow bandwidth due to cyclic prefix (CP) OFDM addition of leakage spectrum because of great side lobes [2], [6], [15], [17]-[19]. Recently, filter bank multi-carrier (FBMC) systems have gained a lot of popularity due to their high bandwidth, power efficiency, flexibility, and spectrum use. Optical FBMC compatible intensity modulation/direct detection (IM/DD) technology [15], [20]. There are several proposed methods for using optical FBMC in VLC systems. Modulation systems must comply with IM/DD [21], [22], meaning that the signal must be real unipolar, so the FBMC modulation system H.S was used to obtain the real signal [23], but this method increases the complexity of IFFT/FFT analysis, where to obtain the number $\mathrm{N}$ symbol $2 \mathrm{~N}$ symbol is used in the frequency field [24], which increases the power consumption, besides that the signal must be unipolar as DC bias is used to convert the bipolar signal to unipolar and this causes power consumption [14], [25]. Modelling the distribution of illumination units in the room is an important factor affecting the distribution of illumination, optical power and SNR [26], [27] The researchers explained many methods of modelling, whether using 4 [28] lighting units or 16 lighting units [29], but these models suffer from low lighting in the middle of the room beside the use of LED with High power consumption and low luminous intensity.

This research, best our knowledge, this the first time that a new scheme for a unipolar FBMC system that does not consume high power is investigated, which is Flip-FBMC, as after the real bipolar signal, the positive part is separated from the negative part, and then the negative part is flipped and made positive and then it is done the real signal is sent positive. Also, a new lighting model was proposed in the room using five lighting units arranged geometrically instead of the traditional model, where the lighting in the centre of the room is less than in the sides, so the new scenario was simulated that covers the inside of the room and the signal reaches the blind area in addition to that it reduces power consumed

\section{DCO-FBMC IN VLC}

FBMC is one of the most promising technologies for $5 \mathrm{G}$ systems, and since VLC is part of $5 \mathrm{G}$, as mentioned above, the real signal must be positive, and for the real signal to be positive without using H.S through the complex signal generated in the classic FBMC system in the time domain, it is of two parts, real and imaginary as in (1) [6], [30].

$$
x_{n}=\sum_{n=-\infty}^{+\infty} \sum_{m=0}^{N-1} a_{m, n} g_{m, n}(t)
$$

Where $n$ is the subcarrier index, $m$ is the time index, $a_{m, n}$ is the massage being transmitted, $N$ is the number of subcarriers; and $g(t)$ is the synthesis function which maps $a_{m, n}$ into the signal space $g_{m, n}(t)$ is the shifted version in time and frequency [14]. After this, the real part is separated from the imaginary part and the imaginary part is converted into real and combined with two consecutively and transmitted, but this real signal is bipolar, so the second condition of the signal is not satisfied, as shown in Figure 1 [23].

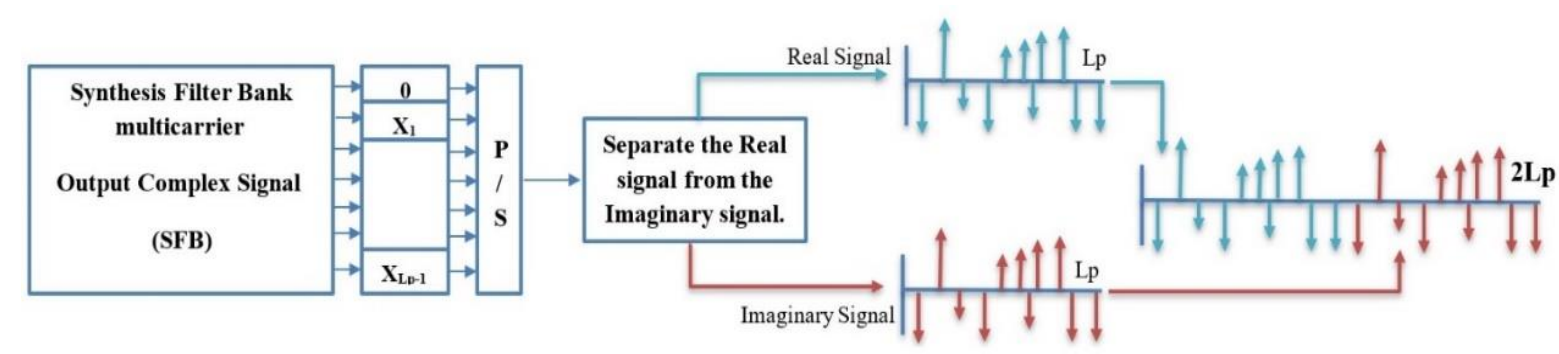

Figure 1. Block diagram of real signal without H.S

The time-domain complex signal can be expressed after passing $\mathrm{P} / \mathrm{S}$ (parallel to serial) as in 2:

$$
x_{n}=a_{n}+j b_{n} \ldots \ldots, n=K(N-1)
$$


Where $a$ and $b$, respectively, represent the real and imaginary part of the complex signal $X_{n}, K$ is the overlapping factor. From here, the length of the pulse modulating filter $(L p)$ can be calculated by $(L p=K N)$.

$$
X_{2 L p}\left\{\begin{array}{cl}
a_{n} & n=0, \ldots . K(N-1) \\
b(n-L p) & n=K N, \ldots .2 K(N-1)
\end{array}\right.
$$

After obtaining the real signal without using H.S by applying the real signal adjacent to the imaginary signal, and thus it is noticed that the signal length is $2 L p$ and the signal will be bipolar as in Figure 2. Since this signal after this stage is a real signal but it is bipolar if it does not comply with IM / DD so it must be positive so DC bias was added to make the signal unipolar.

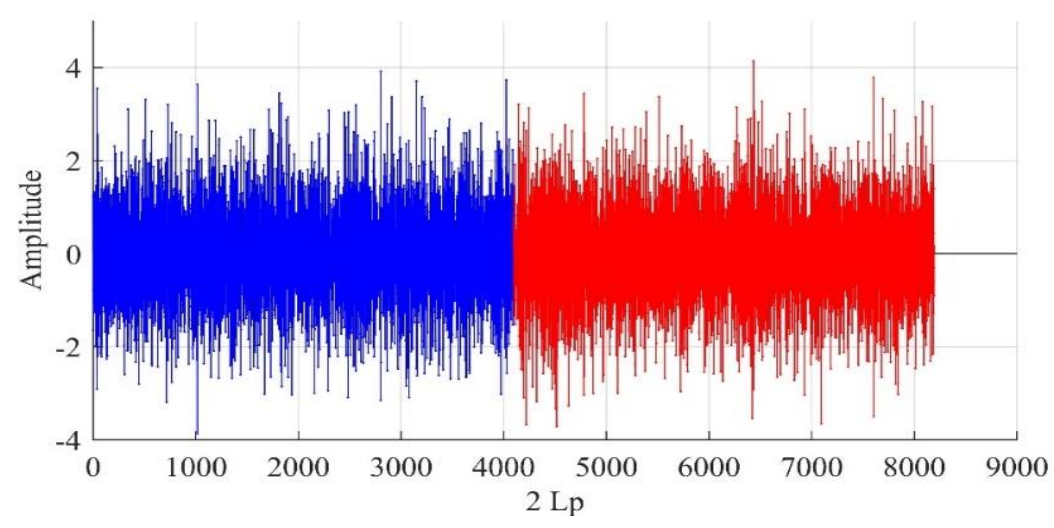

Figure 2. Real signal FBMC without H.S

The negative peaks in the signal must be cut first, so a suitable DC bias is added to the $X_{n}$ signal to eliminate the negative peaks. This adds intermittent noise when the DC bias is high, and this noise decreases affecting the sub-carriers [17].

$$
x_{d c}=X_{n}+B_{d c}+n_{c}
$$

$x_{d c}$ is the DC-bias unipolar signal, $X_{n}$ is real FBMC bipolar signal, $B_{d c}$ and $n_{c}$ are the DC-bias and noise clipping consecutive when the DC-bias becomes large the clipping noise is neglected. the value of DC bias to be set comparable to the power

$$
\begin{aligned}
& B_{d c}=K_{b} \sigma_{n} \\
& K_{b}=\sqrt{10^{R / 10-1}}
\end{aligned}
$$

$K_{b}$ is the clipping factor and $R$ is the bias level in $\mathrm{dB}$, Figure 3 show the DCO-FBMC block diagram.

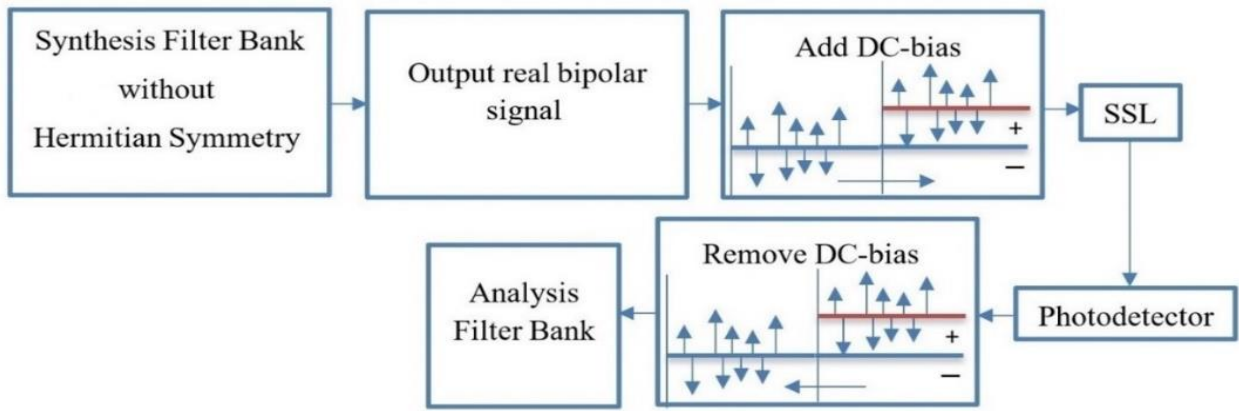

Figure 3. DCO-FBMC block diagram without H.S 


\section{FLIP-FBMC MODULATION}

Optical wireless communication is one of the most important types of communication systems and the VLC system is one of the best types of optical communications as it is inexpensive and has a very wide range of frequencies, so the modulation must be fast and without losses in spectral efficiency or energy consumption. DCO-FBMC is an efficient modulation system that does not suffer from any losses in spectral efficiency, but it suffers from high power consumption, for this reason, it was necessary to find another method that is fast and efficient without losses in spectral efficiency and energy losses.

The Flip-FBMC modulation system is done by separating the positive part from the negative part. The second step is the inverting, the negative part to be positive. As for the third step, the two parts are merged together so that the signal is unipolar and then the real unipolar signal is transmitted to be sent by the optical laser transmitter. Figure 4 shows the block diagram of the Flip-FBMC system from the transmitter to the receiver. As for Figure 5, it shows the shape of the real unipolar signal after leaving the transmitter.

$$
\mathrm{X}_{2 \mathrm{Lp}}=\mathrm{x}_{\mathrm{Lp}}^{+}+\mathrm{X}_{\mathrm{Lp}}^{-}
$$

The real bipolar signal is of length $2 \mathbf{L} \mathbf{p}$ and can be expressed as $\mathbf{x}_{2 \mathbf{L p}}$. As mentioned earlier, the positive $\mathbf{x}^{+} \mathbf{L p}$ part is separated from the negative $\mathbf{x}_{\mathbf{L p}}^{-}$part and they can be defined as follows.

$$
\begin{aligned}
& \mathrm{X}_{\mathrm{Lp}}^{+}=\left\{\begin{array}{l}
\mathrm{x}_{\mathrm{Lp}}^{+} \quad \mathrm{x}_{2 \mathrm{Lp}}>0 \\
\text { otherwise }=0
\end{array}\right. \\
& \mathrm{X}_{\mathrm{Lp}}^{-}=\left\{\begin{array}{c}
\mathrm{X}_{\mathrm{Lp}}^{-} \quad \mathrm{X}_{2 \mathrm{Lp}}<0 \\
\text { otherwise }=0
\end{array}\right.
\end{aligned}
$$

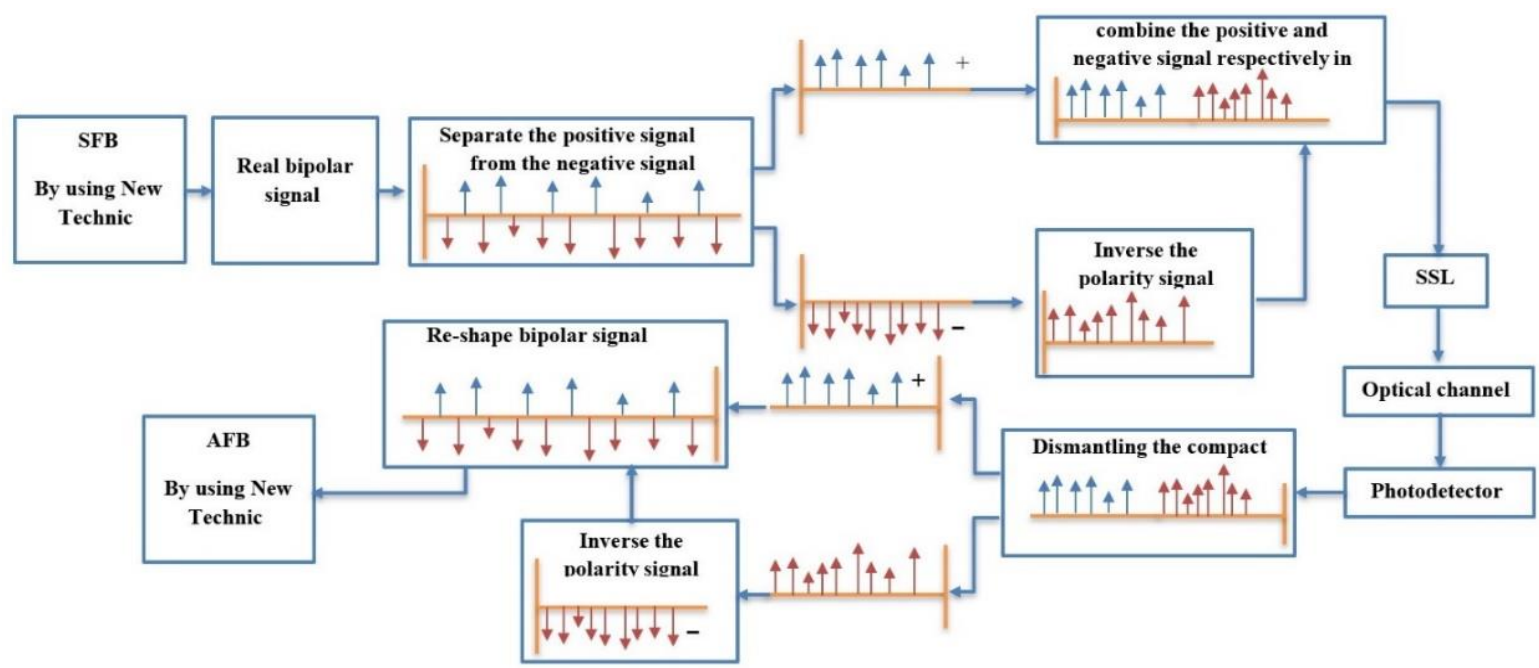

Figure 4. Filp-FBMC block diagram in VLC

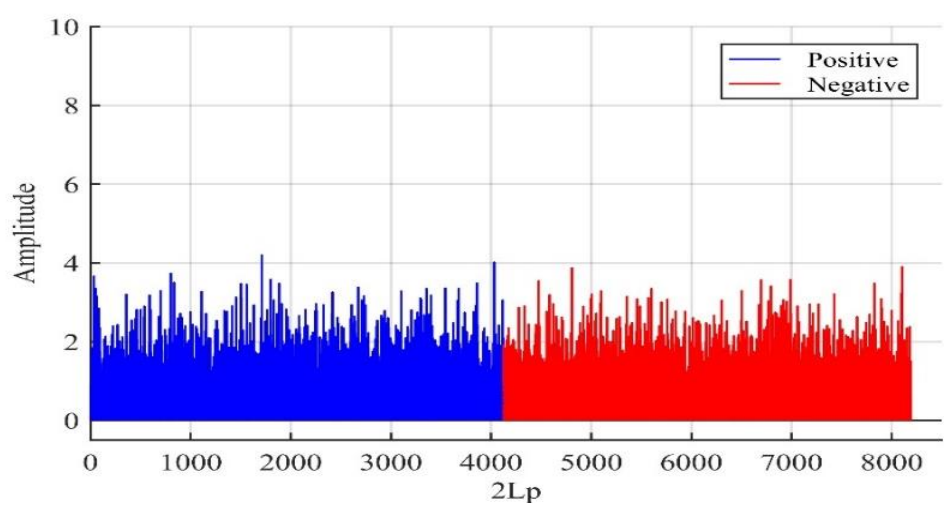

Figure 5. Real unipolar Flip-FBMC signal VLC system 
Since the signal complies with IM/DD, when the optical signal is sent, it is received by the optical detector and converted into an electrical signal, after which it is decoded into two parts. The second part of the positive sign (which is basically negative) is flipped back to its original shape (i.e. negative). After this step, the first part (which is originally positive) is combined with the negative inverted part, and this bipolar signal is restored.

$$
y_{2 L p}=y_{L p}^{+}-y_{L p}^{-}
$$

\section{CHANEL MODEL}

In this research, the effect of semi-angle at half power, field of view (FOV), and the way the lighting is distributed using a laser were also investigated. Table 1 shows the information used in the simulations through which the classic scenario was verified using four light units and the proposed scenario using five modules for the typical room with dimensions of $5 \times 5 \times 3 \mathrm{~m}$ as shown in Figure 6 .

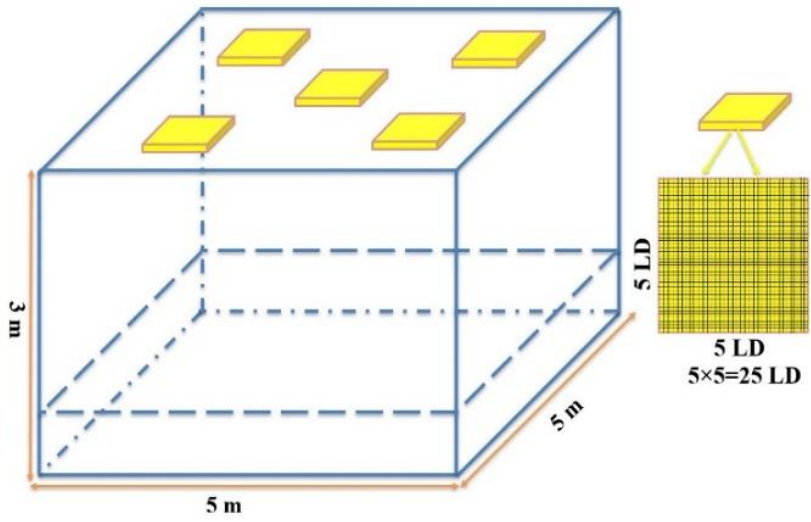

Figure 6. Typical room by using 5 light units

The intensity of radiation in the receiving level can be measured by (11) [9] and after that the luminance is calculated as the light level that the human eye accepts is $300 \mathrm{Lx}$ to $1500 \mathrm{Lx}$ as in (13) as well as calculating the DC gain in (14) [31]-[33].

$$
\begin{aligned}
& I(\partial)=I_{o} \cos ^{m}(\partial) \\
& m=\frac{-\ln 2}{\ln \left(\cos \psi_{c}\right)} \\
& E_{\text {hor }}=\frac{I_{o} \cos ^{m}(\partial)}{D^{2}} \cos (\psi) \\
& H_{(0)}=\left\{\begin{array}{cc}
\frac{(m+1) A}{2 \pi D_{d}^{2}} \cos ^{m}(\partial) T_{s}(\psi) g(\psi) \cos (\psi), 0 \leq \psi \leq \partial_{1 / 2} \\
0, & 0, \psi>\phi_{1 / 2}
\end{array}\right.
\end{aligned}
$$

Where $(\partial)$ is expresses the intensity of the radiation, and so $\mathrm{m}$ is the Lambertian order, $A$ is the optical detector area, $\psi$ is the FOV angle of the photodetector, $T_{s}$ indicates the optical filter gain, $g(\psi)$ is the optical concentrator gain and it is defined by the semi-angle at half the illumination of the laser, as in (12), $E_{\text {hor }}$ represents the level of horizontal illumination as in (13), on the other hand, the total transmitted and received power is calculated as in (15 and 16) respectively [31].

$$
\begin{aligned}
& \mathcal{P}_{c}=\mathcal{P}_{\text {chip }} \times\left[\begin{array}{ccc}
S 1 & \cdots & S 2 \\
\vdots & S 5 & \vdots \\
S 4 & \cdots & S 3
\end{array}\right] \\
& \mathcal{P}_{r}=H_{o} . \mathcal{P}_{c}
\end{aligned}
$$

There is a direct relative relationship between the transmitted optical power and the current of the LD lamp, in which the signal and limitations are described as the optical power. 
The performance of VLC is appropriately evaluated through an evaluation of the signal-to-noise ratio (SNR), which gives the quality of the connection link, and in order to simplify the process, the reflections of the walls are not considered because LoS is dominant in terms of the response of the optical detector [5]. In this way, it is defined as an expression of the quality of communication so the SNR is given as in 17.

$$
S N R=\frac{\left[\mathcal{R} \times \mathcal{P}_{c}\right]^{2}}{\delta_{\text {thermal }}^{2}+\mathcal{S}_{\text {shot }}^{2}}
$$

when the received optical signal strength $\xi$ in the optical communication as in 18:

$$
\xi=\left(\delta_{\text {con }} P_{r(\text { signal })}\right)^{2}
$$

Where the optical to electrical conversion efficiency is $\delta_{\text {con }}$, while the signal intensity in the VLC device is that the time difference between the optical energy of all the contacts reaching the receiver and the first light energy reaching the optical detector is negligible or equal to half the time span of the symbol, the optical power $P_{r(s i g l)}$ can, on the other hand, be measured.

$$
P_{R(s i g)}=\int_{0}^{T}\left[\sum_{m}^{N-1} \sum_{N_{L D=1}^{L \prime s}}^{L D}\left(x_{F l i p} \otimes h_{o}\right) d t\right]
$$

It is therefore possible to determine the SNR of the transmitted Flip-FBMC signal using the following equation.

$$
S N R=\frac{\left[\delta_{\text {con }} \mathcal{R} P_{R(\text { sig })}\right]}{\delta_{\text {thermal }}^{2}+\mathcal{S}_{\text {shot }}^{2}}
$$

Where the detector response is represented by $\mathcal{R}$, add the thermal noise and shot noise representation as $\mathcal{S}_{\text {thermal }}$ and $\mathcal{S}_{\text {shot }}$ respectively [34].

$$
\begin{aligned}
& \mathcal{S}_{\text {shot }}^{2}=2 q \mathcal{R} \mathcal{P}_{c} B+2 q I_{b g} I_{2} B \\
& \mathcal{S}_{\text {thermal }}^{2}=\frac{8 \pi k T_{k}}{G} \eta A I_{2} B^{2}+\frac{16 \pi^{2} k T_{k} \Gamma}{g} \eta^{2} A^{2} I_{3} B^{3}
\end{aligned}
$$

All parameters mentioned in equations is presented in Table 1,

\begin{tabular}{cc} 
Table 1. Simulation parameters \\
\hline Parameters & Values \\
\hline Typical room & $5 \times 5 \times 3 \mathrm{~m}^{3}$ \\
LD unit array & $5 \times 5$ \\
LD $\mathcal{P}_{c}$ & $2 \mathrm{~W}$ \\
$I_{0}$ & $162 \mathrm{~cd}$ \\
$A$ & $1 \mathrm{~cm}^{2}$ \\
$T_{s}$ & 1 \\
$n$ & 1.5 \\
FOV & $40,60,70,80,90$ \\
$\partial$ & $10-70$ \\
c & $3 \times 10^{8} \mathrm{~m} / \mathrm{s}$ \\
$q$ & $1.602 \times 10^{-19} \mathrm{c}$ \\
Data rate & $30 \mathrm{Mb} / \mathrm{s}$ \\
$I_{2}$ & 0.562 \\
$I_{3}$ & 0.0868 \\
$k$ & $1.38066 \mathrm{e}-23$ \\
$T k$ & $295[\mathrm{~K}]$ \\
$G$ & 10 \\
$\Gamma$ & 1.5 \\
$I_{b g}$ & $5100 \mathrm{e}-6$ \\
$\eta$ & $112\left[\mathrm{pF} / \mathrm{cm}^{2}\right]$ \\
$g$ & $30[\mathrm{~ms}]$ \\
$T$ & $10 \mathrm{~ns}$ \\
$\mathcal{R}$ & $0.4 \mathrm{~A} / \mathrm{W}$ \\
\hline
\end{tabular}

The positions of the luminaires in the proposed model are in five units arranged as follows $[(1,1,3)$, $(1,4,3),(4,1,3),(4,4,3),(2.5,2.5,3)]$. 


\section{RESULTS AND DISCUSSION}

The authors in this paper highlight enhance FBMC modulation in a VLC system as well as the proposed luminance model optimization.

\subsection{Flip-FBMC BER performance}

BER is considered one of the most important factors in evaluating the system, as shown in Figure 7, as BER is a function of SNR in $\mathrm{dB}$ unit, although the two techniques Flip-FBMC and DCO-FBMC, although they are of the same length, it was noticed that there is a slight difference in BER that gives preference to the Flip-FBMC technology for different M-OQAM as shown in Figure 7.

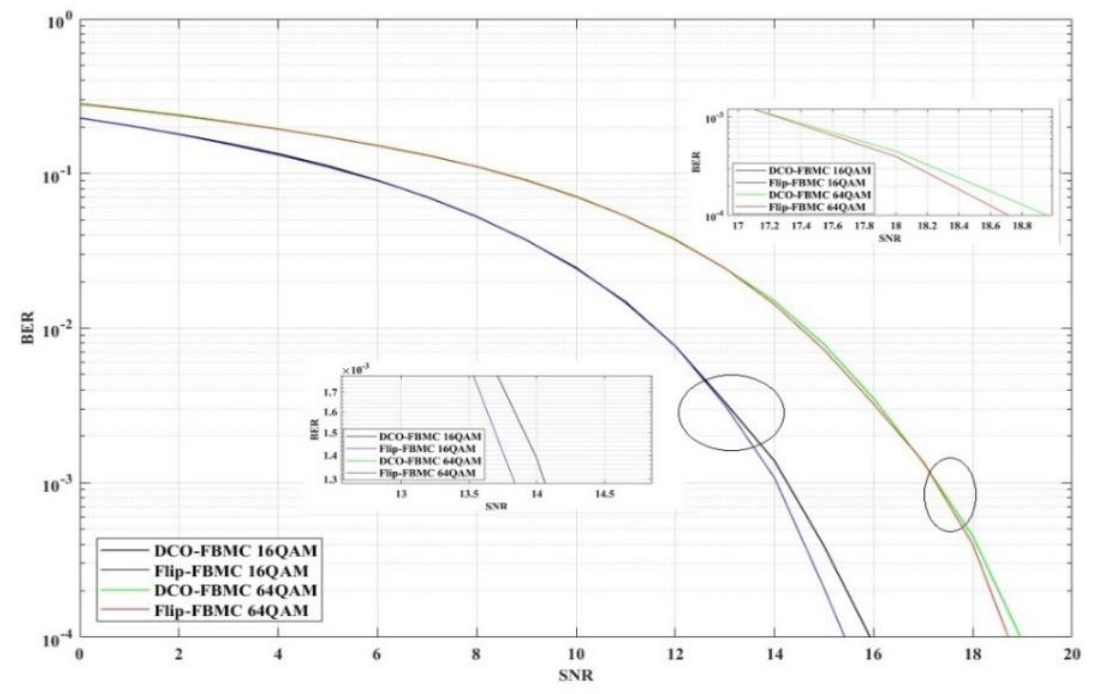

Figure 7. Performance BER of Flip-FBMC vs DCO-FBMC

\subsection{Power consumption unipolar FBMC modulation}

As previously explained, the main reason for suggesting Flip-FBMC technology is to make the signal compatible with IM/DD and reduce the consumed power compared to the power consumed in DCOFBMC technology. Figure 8 shows the average power consumed per M-QAM (4QAM, 16QAM, 64QAM,128 QAM, 256QAM), where Flip-FBMC technology got the lowest power consumed this confirms that Flip-FBMC is more efficient in using power.

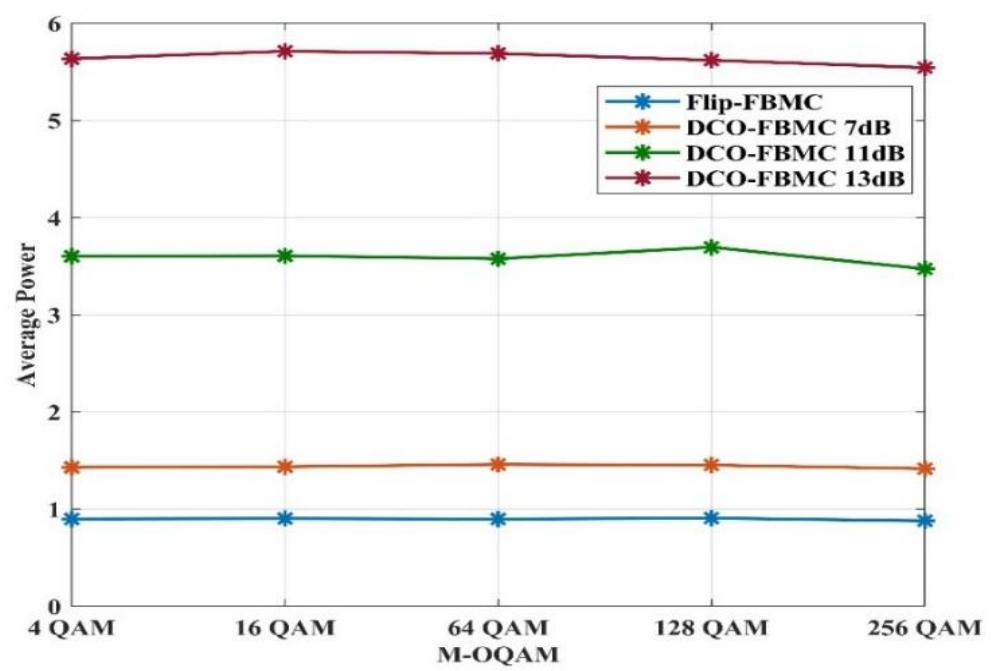

Figure 8. Difference in average power consumption between DCO-FBMC and Flip-FBMC 


\subsection{Horizontal illumination model}

The illumination of the model in the proposed model covers the horizontal area of the ideal room and almost eliminates the blind areas in the room and also making sure that the lighting is within ISO standards. Figure 9 shows the distribution of luminance in the traditional model that uses four luminaires $((1.25,1.25,3),(1.25,3.75,3),(3.75,1.25,3),(3.75,3.75,3))$ [35]-[39], and the proposed model that uses five luminaires, as the figure shows the difference in luminance in the center of the room, as the new model is more preferred as it covers dark areas in different transmitter angles. As for Figure 10, the horizontal lighting shape of the room is displayed for the LD lighting units, as it provides sufficient lighting and covers dark areas in the horizontal room area.

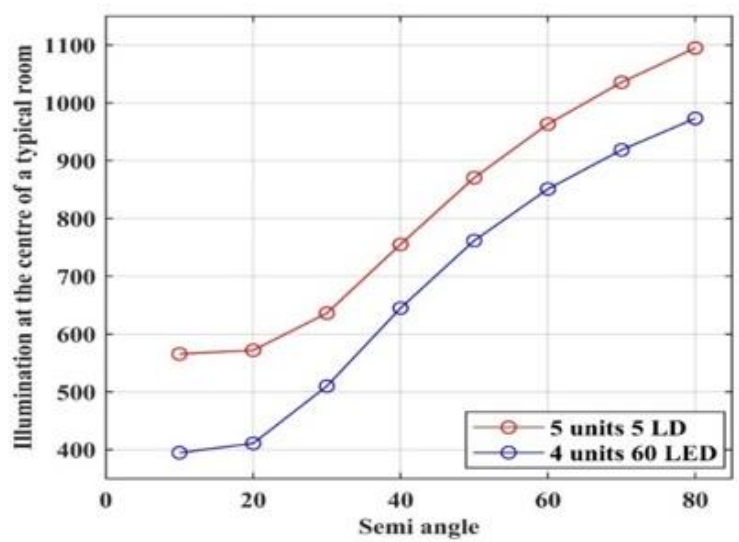

Figure 9. Illuminance distribution in the middle of the room

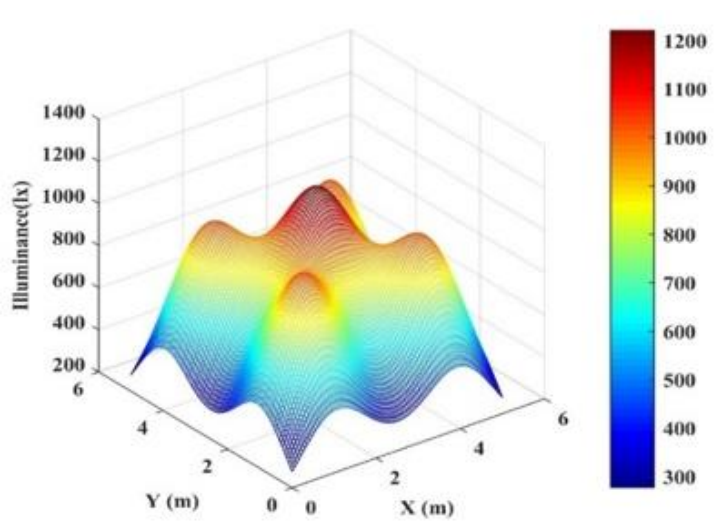

Figure 10. Diatribution horizontal luminance at average lighting $754.76491 x$

\subsection{Optical power}

The proposed model for lighting units contributed really to the case of energy consumption as it consumes less energy than the classic model as the LD units comprise a $5 \times 5$ array for each lighting panel, meaning that the number of LD elements is 125 elements with a total power of $250 \mathrm{~W}$. Unlike the conventional one which consists of four illumination units and each lighting unit consists of $60 \times 60$ LEDs [40], meaning that the entire number of lighting units is 14400 LEDs with a total power of $72 \mathrm{~W}$ [8], and this indicates that the consumption of the traditional model for energy is higher than that of the new model that has been simulated by MATLAB. Figure 11 shows the distribution of the received optical power of the directed radiant light as the rate of the received power at half semi angle 30 is $2.6134 \mathrm{~W}$. Figure 12 shows the relationship between the luminance rate and its correlation with the value of the light power transmitted by the LD light, as it was observed that the increase in brightness leads to an increase in the received light power.

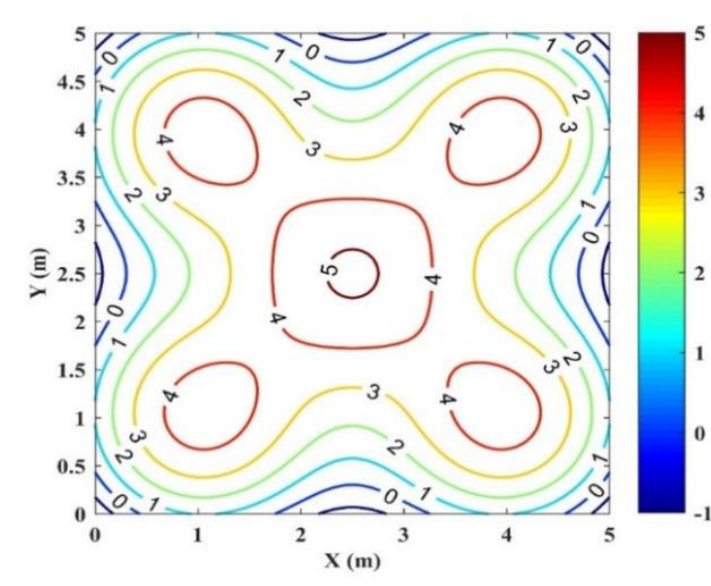

Figure 11. Optical power distribution in the typical room

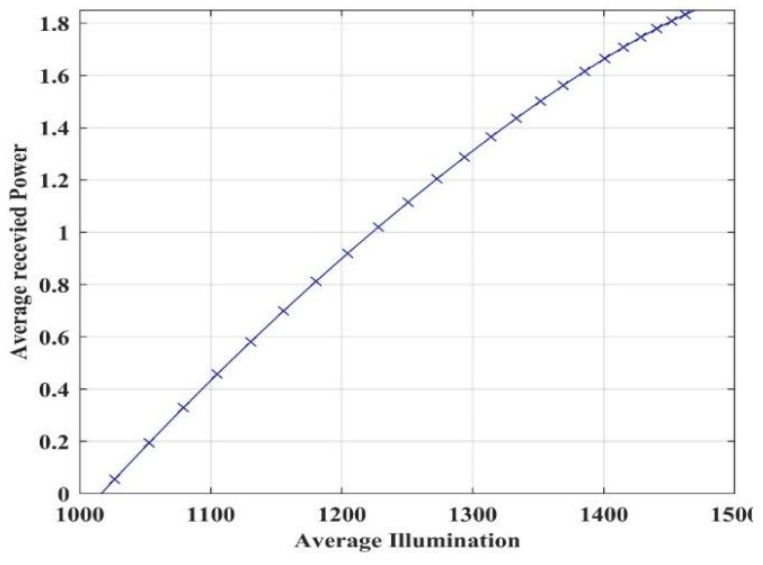

Figure 12. Explains the relationship between luminance and optical power 


\subsection{Signal to noise ratio}

The VLC system includes three main parts of the transmitter source (which includes modulation and optical transmitter (LD)), the transmitter channel and direct detection devices where the system performance is evaluated in terms of the average SNR in the receiver where the SNR values were simulated using FlipFBMC modulation where they were obtained in exchange the transmitted optical power for each LD is as in Figure 13 and Figure 14 shows the distribution of SNR in the typical room. It is observed in Figure 14 that the average relationship of the SNR with the average optical power has an increased relationship, so the higher the optical power value, the more the SNR value.

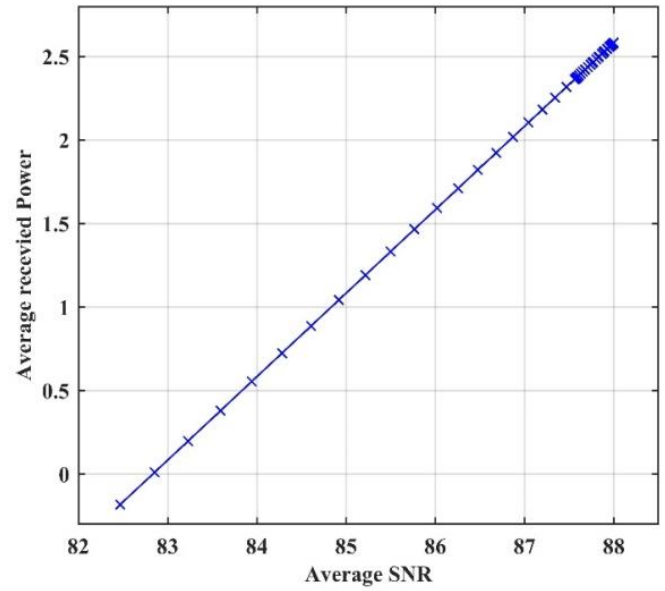

Figure 13. Shown the relationship between average power and average SNR

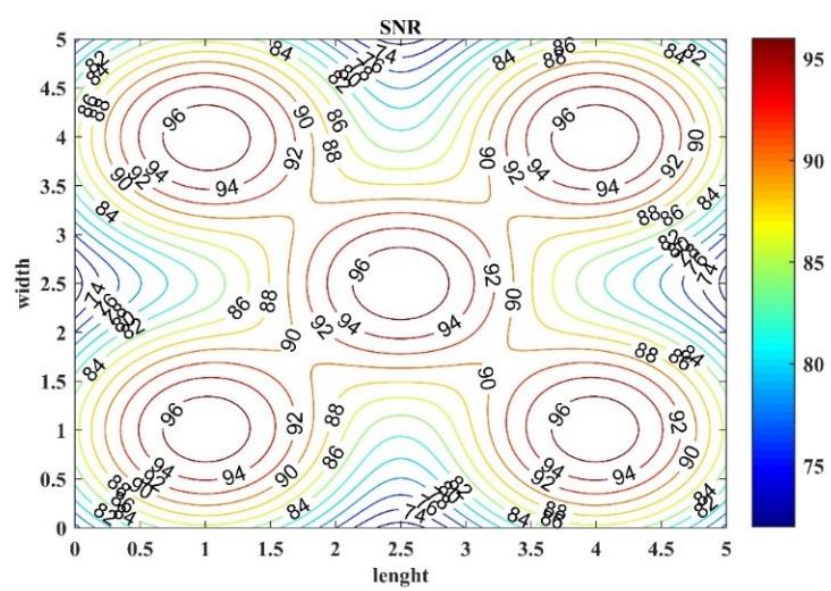

Figure 14. SNR distribution for the typical room at average SNR 88.2278 dB

Figure 15 shows the effect of the half semi angle on the SNR level, as it was tested from 10-70 degree angle, it was observed when the 10 angle was very low and then the SNR value increased at the $20^{\circ}$, but the SNR value decreased as after that it was observed that the SNR level decreased as the half value increased semi angle, where the illuminating circuit is expanded, where it is scattered and is not projected, where the lowest value is recorded at angle $10^{\circ}$ and the highest value at angle $20^{\circ}$.

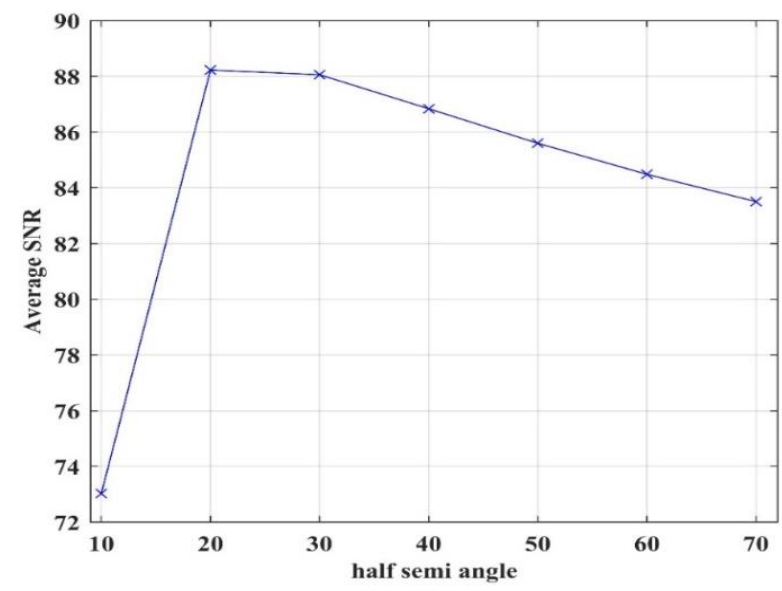

Figure 15. Effect of half semi angle on SNR value

\section{CONCLUSION}

This paper, the authors review the great developments in VLC technology, and at the same time they discussed the improvement of the Flip-FBMC model that was presented in this paper and a comparison with DCO-FBMC technology as the Flip-FBMC technology does not consume additional energy, in addition to it 
gives better BER performance as well. A new illumination distribution model consisting of five lighting units geometrically distributed to eliminate dark areas and provide less energy than the conventional model consumes and deliver the signal to blind areas, where the model gave the best results as the lighting is within ISO, with an average illumination of 754.761lx, the highest optical power 5.1432W, and the maximum SNR 93.1173dB.

\section{REFERENCES}

[1] S. S. Devtale, S. P. Wakle, and R. K. Kulkarni, "Survey Paper on Visible Light," JETIR, vol. 4, no. 06, pp. 216219, 2017.

[2] A. A. Qasim, M. F. L. Abdullah, R. Talib, H. Muwafaq, K. A. Omar, and A. M. Abdulrahman, "Visible Light Communication the next Future Generation System," in 2019 International Conference on Information Science and Communication Technology (ICISCT), 2019, pp. 1-7, doi: 10.1109/CISCT.2019.8777446.

[3] M. S. M. Gismalla, M. F. L. Abdullah, W. A. Mabrouk, B. Das, and M. I. Niass, "Data rate and BER analysis for optical attocells configuration model in visible light communication," in 2019 International Conference on Information Science and Communication Technology, ICISCT 2019, 2019, pp. 1-6, doi: 10.1109/CISCT.2019.8777433.

[4] D. Karunatilaka, F. Zafar, V. Kalavally, and R. Parthiban, "LED based indoor visible light communications: State of the art," IEEE Communications Surveys and Tutorials, vol. 17, no. 3, pp. 1649-1678, 2015, doi: 10.1109/COMST.2015.2417576.

[5] T. Komine and M. Nakagawa, "Fundamental analysis for visible-light communication system using LED lights," IEEE Transactions on Consumer Electronics, vol. 50, no. 1, pp. 100-107, 2004, doi: 10.1109/TCE.2004.1277847.

[6] A. A. Qasim, M. F. L. Abdullah, and R. Talib, "Adaptive DCO-FBMC in Visible Light Communication," IOP Conference Series: Materials Science and Engineering, vol. 767, no. 1, 2020, doi: 10.1088/1757899X/767/1/012018.

[7] H. Chun, S. Rajbhandari, D. Tsonev, G. Faulkner, H. Haas, and D. O’Brien, "Visible light communication using laser diode based remote phosphor technique," in 2015 IEEE International Conference on Communication Workshop, ICCW 2015, 2015, pp. 1392-1397, doi: 10.1109/ICCW.2015.7247373.

[8] M. T. Niaz, F. Imdad, S. Kim, and H. S. Kim, "Deployment methods of visible light communication lights for energy efficient buildings," Optical Engineering, vol. 55, no. 10, p. 106113, 2016, doi: 10.1117/1.OE.55.10.106113.

[9] M. S. M. Gismalla, M. F. L. Abdullah, M. I. Niass, B. Das, and W. A. Mabrouk, "Improve uniformity for an indoor visible light communication system," International Journal of Communication Systems, vol. 33, no. 8, pp. 1-13, 2020, doi: $10.1002 /$ dac.4349.

[10] M. T. Niaz, F. Imdad, W. Ejaz, and H. S. Kim, "Compressed sensing-based channel estimation for ACO-OFDM visible light communications in 5G systems," Eurasip Journal on Wireless Communications and Networking, vol. 2016, no. 1, pp. 1-14, 2016, doi: 10.1186/s13638-016-0774-2.

[11] Z. Wang and Q. Wang, "Optical OFDM for visible light communications," in IEEE 13th International Wireless Communications and Mobile Computing Conference (IWCMC), vol. 1, no. 1, 2017, pp. 4546, doi: 10.1109/IWCMC.2017.7986454.

[12] S. K. Hashemi, Z. Ghassemlooy, L. Chao, and D. Benhaddou, "Orthogonal frequency Division Multiplexing for indoor optical wireless communications using visible light LEDs," in Proceedings of the 6th International Symposium Communication Systems, Networks and Digital Signal Processing, CSNDSP 08, pp. 174-178, 2008, doi: 10.1109/CSNDSP.2008.4610804.

[13] J. Armstrong and B. J. C. Schmidt, "Comparison of asymmetrically clipped optical OFDM and DC-biased optical OFDM in AWGN," IEEE Communications Letters, vol. 12, no. 5, pp. 343-345, 2008, doi: 10.1109/LCOMM.2008.080193.

[14] A. A. Qasim, M. F. L. Abdullah, H. N. Mohammedali, R. Bin Talib, M. N. Nemah, and A. T. Hammoodi, "Low complexity DCO-FBMC visible light communication system," International Journal of Electrical and Computer Engineering, vol. 10, no. 1, pp. 928-934, 2020, doi: 10.11591/ijece.v10i1.pp928-934.

[15] S. Niu, P. Wang, S. Chi, Z. Liu, W. Pang, and L. Guo, "Enhanced Optical OFDM/OQAM for Visible Light Communication Systems," IEEE Wireless Communications Letters, vol. 2337, no. c, 2020, doi: 10.1109/LWC.2020.3040178.

[16] A. A. Abdulkafi, M. Y. Alias, and Y. S. Hussein, "Performance analysis of DCO-OFDM in VLC system," in 2015 IEEE 12th Malaysia International Conference on Communications, MICC 2015, vol. 1, no. 12, 2015, pp. 163-168, doi: 10.1109/MICC.2015.7725427.

[17] J. Armstrong, B. J. C. Schmidt, D. Kalra, H. A. Suraweera, and A. J. Lowery, "Performance of asymmetrically clipped optical OFDM in AWGN for an intensity modulated direct detection system," in GLOBECOM - IEEE Global Telecommunications Conference, no. November, 2006, pp. 2-7, doi: 10.1109/GLOCOM.2006.571.

[18] H. Kazemi and H. Haas, "On the performance of single side-band OFDM for band-limited visible light communication," 2020 IEEE International Conference on Communications Workshops, ICC Workshops 2020 Proceedings, 2020, doi: 10.1109/ICCWorkshops49005.2020.9145204.

[19] R. Hassan and F. T. Z. Tuli, "Analysis of ACO-OFDM, DCO-OFDM and Flip-OFDM for IM/DD optical-wireless and optical-fiber system," in 1st IEEE International Conference on Telecommunications and Photonics, ICTP 2015, 2016, no. December 2014, pp. 1-5, doi: 10.1109/ICTP.2015.7427927.

[20] B. Lin et al., "Experimental Demonstration of OFDM/OQAM Transmission for Visible Light Communications," IEEE Photonics Journal, vol. 8, no. 5, pp. 1-10, 2016, doi: 10.1109/JPHOT.2016.2605464. 
[21] R. Chen, K. H. Park, C. Shen, T. K. Ng, B. S. Ooi, and M. S. Alouini, "Visible light communication using DCbiased optical filter bank multi-carrier modulation," in 2018 Global LIFI Congress, GLC 2018, vol. 2018-Janua, 2018, pp. 1-6, doi: 10.23919/GLC.2018.8319094.

[22] M. S. M. Gismalla, M. F. Abdullah, M. Sami, N. S. M. Shah, B. Das, and A. A. Qasim, "Effect of Optical Attocells Deployment on the RMSD Spread in Indoor Visible Light Communication Systems," 2020 International Conference on Information Science and Communication Technology, 2020, pp. 1-6, doi: 10.1109/ICISCT49550.2020.9079939.

[23] A. A. Qasim, M. F. L. Abdullah, and R. Talib, "Adaptive DCO-FBMC in Visible Light Communication," in IOP Conference Series: Materials Science and Engineering, vol. 767, no. 1, 2020, doi: 10.1088/1757899X/767/1/012018.

[24] M. El Tabach, P. Tortelier, R. Pyndiah, and O. Bouchet, "Diffuse Infrared Personal optical wireless based on modified OFDM/OQAM," in Proceedings of the 6th International Symposium Communication Systems, Networks and Digital Signal Processing, CSNDSP 08, no. 2, pp. 161-164, 2008, doi: 10.1109/CSNDSP.2008.4610752.

[25] M. M. Mijwil, "High speed transmission of signal level for white light emitting diode (LED) as a transmitter device by using modified phase equalization," Indonesian Journal of Electrical Engineering and Computer Science, vol. 17, no. 3, pp. 1348-1354, 2019.

[26] H. Q. Nguyenl, J. C. M. Kang, Z. G, D. H. Kim, C. G. Lee, and S. Lim, “A MA TLAB-based simulation program for indoor visible light communication system," in CSNDSP, 2010, pp. 537-541, doi: 10.1109/CSNDSP16145.2010.5580355.

[27] D. Ding, X. Ke, and L. Xu, "An optimal lights layout scheme for visible-light communication system," in 2007 8th International Conference on Electronic Measurement and Instruments, ICEMI, 2007, pp. 2189-2194, doi: 10.1109/ICEMI.2007.4350650.

[28] T. H. Do and M. Yoo, "Optimization for link quality and power consumption of visible light communication system," Photonic Network Communications, vol. 27, no. 3, pp. 99-105, 2014, doi: 10.1007/s11107-014-0430-x.

[29] N. E. Mahfouz, H. A. Fayed, A. Abd El Aziz, and M. H. Aly, "Improved light uniformity and SNR employing new LED distribution pattern for indoor applications in VLC system," Optical and Quantum Electronics, vol. 50, no. 9, pp. 1-8, 2018, doi: 10.1007/s11082-018-1618-6.

[30] S. Kumar and P. Singh, "Spectral Efficient Asymmetrically Clipped Hybrid FBMC for Visible Light Communication," International Journal of Optics, vol. 2021, pp. 1-8, 2021, doi: 10.1155/2021/8897928.

[31] A. A. Qasim, M. F. L. Abdullah, Q. J. Kadhim, R. Talib, A. M. Alsahlany, and M. S. M. Gismalla, "Modelling 5 Units Illumination for Visible Light Communication System," in ICISCT 2020 - 2nd International Conference on Information Science and Communication Technology, 2020, doi: 10.1109/ICISCT49550.2020.9079946.

[32] Z. Ghassemlooy, D. Wu, M.-A. Khalighi, and X. Tang, "Indoor non-directed optical wireless communications Optimization of the Lambertian order," Journal of Electrical and Computer Engineering Innovations, vol. 1, no. 1, pp. 1-9, 2013.

[33] T. Komine, J. H. Lee, S. Haruyama, and M. Nakagawa, "Adaptive equalization system for visible light wireless communication utilizing multiple white led lighting equipment," IEEE Transactions on Wireless Communications, vol. 8, no. 6, pp. 2892-2900, 2009, doi: 10.1109/TWC.2009.060258.

[34] T. C. Bui, S. Kiravittaya, N. H. Nguyen, N. T. Nguyen, and K. Spirinmanwat, "LEDs configuration method for supporting handover in visible light communication," IEEE Region 10 Annual International Conference, Proceedings/TENCON, vol. 2015-Janua, no. Vlc, 2015, pp. 1-6, doi: 10.1109/TENCON.2014.7022432.

[35] H. Q. Nguyen, J. H. Choi, M. Kang, Z. Ghassemlooy, and D. H. Kim, "A MATLAB-based simulation program for indoor visible light communication system," in CSNDSP, 2010, no. June 2014, p. Newcastle upon Tyne, UK, doi: 10.1109/CSNDSP16145.2010.5580355.

[36] D. Tronghop, J. Hwang, S. Jung, Y. Shin, and M. Yoo, "Modeling and analysis of the wireless channel formed by LED angle in visible light communication," in International Conference on Information Networking, no. 2010, 2012, pp. 354-357, doi: 10.1109/ICOIN.2012.6164399.

[37] R. Guan, J. Y. Wang, Y. P. Wen, J. B. Wang, and M. Chen, "PSO-based LED deployment optimization for visible light communications," 2013 International Conference on Wireless Communications and Signal Processing, WCSP 2013, no. 1, 2013, pp. 1-6, doi: 10.1109/WCSP.2013.6677122.

[38] M. Irshad, W. Liu, M. N. Sohail, D. Qaiser, M. M. Uba, and M. M. Bilal, "VLC based LOS \& Non LOS propagation model," International Journal of Advanced Research in Computer and Communication Engineering, vol. 7, no. 10, pp. 1-6, 2018.

[39] B. Fahs, A. J. Chowdhury, and M. M. Hella, "A 12-m 2 . 5-Gb/s Lighting Compatible Integrated Receiver for OOK Visible Light Communication Links," Journal of Lightwave Technology, vol. 34, no. 16, pp. 3768-3775, 2016, doi: 10.1109/JLT.2016.2587598.

[40] I. Din and H. Kim, "Energy-efficient brightness control and data transmission for visible light communication," IEEE Photonics Technology Letters, vol. 26, no. 8, pp. 781-784, 2014, doi: 10.1109/LPT.2014.2306195. 